\title{
Detecção Precoce das Doenças de Alzheimer e Parkinson através de Parâmetros Não-Lineares Multibanda de Sinais EEG
}

\author{
Gabriel A. R. Silva, Marco A. R. Alves, Bruno C. Bispo e Pedro M. Rodrigues
}

\begin{abstract}
Resumo-Este trabalho tem como objetivo a detecção precoce das doenças de Alzheimer e Parkinson através de parâmetros não-lineares multibanda de sinais EEG. Para cada par de grupos de estudo, uma seleção dos parâmetros é realizada através de algoritmo genético. Os parâmetros selecionados são utilizados como entrada para classificadores com validação cruzada leaveone-out. Acurácias de classificação de $100 \%$ são obtidas, em pelo menos uma sub-banda, para 3 pares de grupos de estudo enquanto $90,60 \%$ é alcançado para o par Controle vs Alzheimer/Parkinson. A sub-banda delta foi a que, em geral, apresentou maiores diferenças significativas entre os grupos.
\end{abstract}

Palavras-Chave-Detecção precoce, Alzheimer, Parkinson, EEG, análise não-linear, wavelet.

Abstract-This work aims to detect Alzheimer's and Parkinson's diseases at early stage through non-linear multiband parameters of EEG signals. For each pair of study groups, parameters selection was performed through genetic algorithm. The selected parameters are used as input for classifiers with leave-one-out cross-validation. Classification accuracies of $100 \%$ are achieved, in at least one subband, for 3 pairs of study groups while 90.60\% is achieved for the Control vs Alzheimer/Parkinson pair The delta subband showed, in general, the greatest significant differences between the groups.

Keywords-Early detection, Alzheimer, Parkinson, EEG, nonlinear analysis, wavelet.

\section{INTRODUÇÃo}

Entre as doenças neurodegenerativas, as doenças de Alzheimer (DA) e Parkinson (DP) destacam-se por vários fatores: apresentam as maiores incidências a nível mundial [1], [2]; possuem períodos assintomáticos, [3], [4]; as causas não são conhecidas com exatidão [5]; e, apesar de existirem soluções que permitem atrasar o seu avanço, não há cura [6], [7]. Portanto, a pesquisa por métodos para detectar precocemente essas doenças é de extrema importância para retardar a progressão e os sintomas das mesmas, contribuindo assim para uma melhor qualidade e maior expectativa de vida dos pacientes.

A evolução da DA e DP são divididas em estágios com diferentes escalas. No entanto, um estágio pré-doença, denominado défice cognitivo leve (MCI, do inglês Mild Cognitive Impairment), é normalmente considerado como o estágio inicial de todas as doenças neurodegenerativas, sendo a transição entre o envelhecimento normal e a patologia. Nesta fase, os sintomas são pouco evidentes, normalmente lapsos esporádicos de memória, e entendidos pelo paciente como uma consequência normal da idade ou atribuídos ao cansaço [8]. Essa fase pode ou não evoluir para a doença em si.

Gabriel A. R. Silva, Marco A. R. Alves e Pedro M. Rodrigues, Universidade Católica Portuguesa, CBQF - Centro de Biotecnologia e Química Fina Laboratório Associado, Escola Superior de Biotecnologia, Porto, Portugal, email: prodrigues@porto.ucp.pt; Bruno C. Bispo, Departamento de Engenharia Elétrica e Eletrônica, Universidade Federal de Santa Catarina, FlorianópolisSC, Brasil, e-mail: bruno.bispo@ufsc.br.
Os ambientes clínicos costumam utilizar exames que medem a capacidade física e cognitiva dos pacientes para avaliar a evolução dessas doenças. O mais comum é o mini exame do estado mental (MMSE, do inglês Mini Mental State Exam), que consiste de perguntas para avaliar a capacidade de orientação, atenção, memória e linguagem do paciente [9].

Entre as diversas ferramentas médicas disponíveis para avaliar marcadores biológicos relacionados a essas doenças, como ressonância magnética, tomografia por emissão de pósitrons, tomografia computadorizada por emissão de fóton único, magnetoencefalografia, análise de sangue e do fluído cerebrospinal, a eletroencefalografia (EEG) destaca-se por ser amplamente utilizada em ambientes clínicos devido ao seu sistema de gravação ser barato, não-invasivo, móvel e rápido.

Alguns trabalhos sobre detecção de DA e DP em estágio inicial através de sinais EEG são encontrados na literatura. Em relação à discriminação entre indivíduos saudáveis e pacientes com MCI que evoluíram para DP, uma acurácia de $82 \%$ foi alcançada em [10] ao aplicar algumas métricas espectrais e sete fatores temporais extraídos por meio de análise de componentes principais a máquinas de vetores suporte (SVM, do inglês Support Vector Machine). Em [11], uma rede neural convolucional foi proposta para aprender características de alto nível dos sinais, obtendo uma acurácia de $88,25 \%$.

$\mathrm{Na}$ discriminação entre indivíduos saudáveis e pacientes com MCI que evoluíram para DA, uma acurácia de $93 \%$ foi obtida em [12] ao aplicar parâmetros de um método de modelagem de colisão a uma rede neural artificial (RNA). Em [13], características temporais extraídas do espectro foram aplicadas a SVM, árvores de decisão e técnicas de mineração de dados, alcançando uma acurácia de 90\%. Em [14], a combinação de características que representavam a desaceleração dos sinais e RNA resultou numa acurácia de 77\%. Em [15], uma seleção de 25 características temporais e espectrais de sinais EEG, incluindo potenciais relacionados a eventos, foram aplicados a SVM, alcançando uma classificação de $88 \%$.

Esse trabalho propõe detectar DA e DP em estágio inicial através de parâmetros não-lineares de sinais EEG. O interesse pela análise não-linear de sinais EEG advém da sua própria origem, uma vez que o cérebro é um sistema complexo dependente de inúmeros fenômenos biológicos e fisiológicos cujo comportamento é não-linear [16]. E com intuito de explorar uma possível maior capacidade discriminante de bandas frequenciais, os parâmetros serão obtidos a partir de uma análise multibanda através da transformada Wavelet. $\mathrm{O}$ artigo está organizado da seguinte maneira: a Seção II descreve a base de EEG utilizada; a Seção III explica a decomposição multibanda realizada; a Seção IV apresenta os parâmetros nãolineares utilizados; a Seção V apresenta e discute os resultados obtidos; e, por fim, a Seção VI conclui o trabalho. 
TABELA I

INFORMAÇÃO DA BASE DE DADOS.

\begin{tabular}{c|c|c|c}
\hline Grupo & No de indivíduos & Idade média & MMSE \\
\hline \hline Controle & 16 & 74 & 28,68 \\
DP (MCI) & 8 & 70 & 28,64 \\
DA (MCI) & 8 & 80 & 26,29 \\
\hline
\end{tabular}

\section{BASE DE DADOS DE EEG}

A base de dados consiste de 32 exames de EEG gravados no Hospital de São João, Porto, Portugal, projeto CES198-14, com a aprovação do comitê de ética local e do conselho de administração do hospital. Os sinais foram adquiridos a uma frequência de amostragem de $256 \mathrm{~Hz}$ através de 19 eletrodos colocados sobre o escalpe de acordo com o sistema 10-20, resultando em 19 sinais por exame (indivíduo). Os indivíduos encontravam-se num estado de relaxamento e com os olhos fechados. Entre eles, 16 são saudáveis (controle), 8 diagnosticados com MCI que evoluiu para DA e 8 diagnosticados com MCI que evoluiu para DP. O MMSE e a idade média dos grupos de estudo estão apresentados na Tabela I.

Todos os sinais foram filtrados usando um filtro passa-banda digital com frequências de corte de 1 e $40 \mathrm{~Hz}$, faixa frequencial convencionais de EEG, a fim de remover artefatos e ruído elétrico. A amplitude de cada sinal foi normalizada segundo

$$
x(n)=\frac{x(n)}{\sum_{n=0}^{N-1} x^{2}(n)},
$$

onde $N$ representa o tamanho dos sinais, e a componente contínua foi removida. E cada sinal foi segmentado em 20 trechos não-sobrepostos com duração de $5 \mathrm{~s}$. Para cada indivíduo, criou-se um segmento médio ao realizar a média dos 20 segmentos dos seus 19 canais.

\section{DeComposiçÃo MultibandA}

A transformada wavelet discreta (DWT, do inglês Discrete Wavelet Transform) de um sinal de tempo discreto e energia finita é a sua decomposição em um conjunto de funções base obtidas a partir de um número finito de sequências protótipas e suas versões deslocadas no tempo [17]. Essa expansão estruturada é implementada por meio de um banco de filtros em bandas de oitavas e criticamente decimado [17], [18].

A DWT utiliza uma função escala de análise $\tilde{\phi}_{1}(n)$ e uma função wavelet de análise $\psi_{1}(n)$ que são respostas ao impulso dos filtros de análise passa-baixa e passa-alta de meia banda, respectivamente. Definindo as seguintes fórmulas de recursão

$$
\begin{gathered}
\tilde{\phi}_{i+1}(n)=\tilde{\phi}_{i}(n / 2) * \tilde{\phi}_{1}(n) \\
\tilde{\psi}_{i+1}(n)=\tilde{\phi}_{i}(n) * \tilde{\psi}_{1}\left(n / 2^{i}\right),
\end{gathered}
$$

o filtro de análise equivalente da $m$-ésima sub-banda é dado por

$$
h_{m}(n)= \begin{cases}\tilde{\phi}_{S}(n), & m=0 \\ \tilde{\psi}_{S+1-m}(n), & m=1,2, \ldots, S .\end{cases}
$$

E o sinal da $m$-ésima sub-banda do sinal $x(n)$ é dado por

$$
x_{m}(n)= \begin{cases}\sum_{k=-\infty}^{\infty} x(k) h_{m}\left(2^{S} n-k\right), & m=0, \\ \sum_{k=-\infty}^{\infty} x(k) h_{m}\left(2^{S-m+1} n-k\right), & m=1,2, \ldots, S .\end{cases}
$$

Neste trabalho, a DWT foi aplicada aos 20 segmentos médios de cada indivíduo com o intuito de decompô-los nas sub-bandas convencionais de EEG, nomeadamente, $\delta(1-4 \mathrm{~Hz})$, $\theta(4-8 \mathrm{~Hz}), \alpha(8-13 \mathrm{~Hz}), \beta(13-30 \mathrm{~Hz})$ e $\gamma(30-40 \mathrm{~Hz})$. Neste caso, $S=5$ e os sinas das sub-bandas se relacionam com as sub-bandas de EEG da seguinte forma: $x_{\delta}(n)=x_{0}(n)$, $x_{\theta}(n)=x_{1}(n), x_{\alpha}(n)=x_{2}(n), x_{\beta}(n)=x_{3}(n)$ e $x_{\gamma}(n)=$ $x_{4}(n)$. O sinal $x_{5}(n)$ não é utilizado. Os sinais das sub-bandas foram reamostrados para a frequência de amostragem original através do método de interpolação wavelet [19].

\section{ANÁLISE NÃO-LINEAR}

A natureza não-linear dos sinais EEG da base de dados foi verificada por meio da ferramenta disponibilizada em [20]. Doravante, o sinal $x(n)$ possui tamanho $N$ e média $\bar{x}$.

\section{A. Medidas do Atrator Reconstruído}

Em cada instante de tempo, o estado de um sistema dinâmico definido por $m$ variáveis pode ser representado por um ponto no espaço $m$-dimensional. Nesse espaço, denominado espaço de estado ou de fase, a sequência de estados ao longo do tempo define curvas denominadas trajetórias. Estas trajetórias, quando observadas por longos períodos, podem convergir para uma estrutura geométrica específica e independente das condições inicias do sistema, denominada atrator [21].

Entre as técnicas para representar o espaço de estado de um sistema dinâmico a partir de uma única série temporal (sinal), processo denominado reconstrução, a técnica de incorporação de atraso é a mais utilizada para sinais EEG [21]. Nesse caso, uma reconstrução do espaço de estado é dada por [21]

$$
\mathbf{x}_{i}=[x(i), x(i+\tau), \ldots, x(i+(m-1) \tau)],
$$

onde $\tau$ é o atraso de incorporação. Os valores de $\tau$ e $m$ foram obtidos de $x(n)$ por meio dos métodos descritos em [21], [22].

A sequência de vetores $\mathbf{x}_{i}, i=1,2, \ldots, M$ onde $M=$ $N-(m-1) \tau$, formam o atrator reconstruído.

1) Dimensão da Correlação: Medida que caracteriza a distribuição dos pontos do atrator, refletindo a complexidade do sistema dinâmico, estimada como [21], [22]

$$
D_{2}=\lim _{r \rightarrow 0} \frac{\log (C(r, M))}{\log (r)},
$$

onde

$$
C(r, M)=\frac{2}{M(M-1)} \sum_{i=1}^{M} \sum_{\substack{j=1 \\ j \neq i}}^{M} \Theta\left(r-\left\|\mathbf{x}_{i}-\mathbf{x}_{j}\right\|\right)
$$

é a probabilidade do par de pontos $\left\{\mathbf{x}_{i}, \mathbf{x}_{j}\right\}$ no atrator estar separado por uma distância menor ou igual a $r$ e $\Theta$ é a função Heaviside [21], [22].

2) Expoentes de Lyapunov: Medidas que fornecem informação sobre a evolução das trajetórias ao longo do tempo [22], refletindo a estabilidade do sistema dinâmico [21]. Coeficientes positivos e negativos indicam divergência e convergência exponencial de trajetórias próximas no atrator, respectivamente [23]. Um sistema caótico tem pelo menos um expoente positivo [22], [23]. O maior expoente de Lyapunov (LLE, do inglês Largest Lyapunov Exponent) pode ser estimado ao, para cada estado $\mathbf{x}_{i}$, encontrar o estado $\mathbf{x}_{j}$ que satisfaça $\min _{j}\left\|\mathbf{x}_{i}-\mathbf{x}_{j}\right\|$ tal que $|i-j|>T_{m}$, onde $T_{m}$ é o período médio de $x(n)$. 
As estimativas são dadas por [24]

$$
\lambda(i)=\frac{1}{M+2} \sum_{k=1}^{M} \frac{1}{k * T_{s}} \ln \frac{\left\|\mathbf{x}_{i+k}-\mathbf{x}_{j+k}\right\|}{\left\|\mathbf{x}_{i}-\mathbf{x}_{j}\right\|},
$$

onde $T_{s}$ é o período de amostragem. E o LLE é definido pela inclinação da melhor aproximação linear de $\lambda(i)$ [24].

\section{B. Medidas de Memória Longa}

1) Expoente Hurst: Medida da dependência estatística de longo alcance de uma série temporal, utilizada para avaliar a sua suavidade, propriedades de correlação e grau de autosimilaridade [21], [22]. Pode ser definida como [22]

$$
H=\frac{\log (R / S)}{\log (N)},
$$

onde $R / S$ é uma medida estatística denominada intervalo reescalonado. Na prática, $H$ é estimado pela inclinação da melhor aproximação linear de $\log [R(n) / S(n)]$ em função de $\log (n)$, onde detalhes sobre o cálculo de $R(n) / S(n)$ podem ser encontrados em [25]. Quanto mais irregular o sinal de EEG, mais próximo de 0 estará $H$ [21].

2) Análise de Flutuação Destendenciada: Método de análise que fornece um parâmetro para quantificar as correlações de longo alcance (auto-similaridade) de séries temporais aparentemente não-estacionárias [26], [27]. A partir de $x(n)$, calcula-se a série de desvio cumulativo $y(k)=$ $\sum_{i=1}^{k}[x(i)-\bar{x}]$. Em seguida, para cada trecho de comprimento $m$ de $y(k)$, calcula-se uma aproximação linear denotada por $y_{m}(k)$. A flutuação média do sinal em função de $m$ é definida como

$$
F(m)=\sqrt{\frac{1}{N} \sum_{k=1}^{N}\left[y(k)-y_{m}(k)\right]^{2}} .
$$

A inclinação da melhor aproximação linear de $\log [F(m)]$ em função de $\log (m)$ é o expoente de escala $\alpha$ que representa as propriedades de correlação do sinal $x(n)$ [27].

\section{Dimensão Fractal}

A dimensão fractal (FD, do inglês Fractal Dimension) de um objeto geométrico é uma medida de sua complexidade - extensão espacial, complicação ou propensão ao preenchimento do espaço - e está intimamente ligada à sua forma [28].

Numa forma de onda, a FD é uma ferramenta poderosa para a detecção de eventos transitórios [22]. Ela pode ser calculada diretamente a partir dos sinais, não havendo necessidade de reconstruir o atrator. Entre os algoritmos para determinar a FD de uma forma de onda, os brevemente descritos abaixo são conhecidos por serem adequados para análise de EEG [22].

1) Algoritmo de Higuchi: Para $m=1, \ldots, k$ e $k=$ $1, \ldots, k_{\max }$, onde $k_{\max }$ é obtido experimentalmente apesar de $k_{\max }=8$ ter sido inicialmente proposto, calcula-se a seguinte medida de distância [21], [22]

$$
L_{m}(k)=\frac{N-1}{\lfloor a\rfloor k} \sum_{i=1}^{\lfloor a\rfloor}|x(m+i k)-x(m+(i-1) k)|,
$$

onde $a=(N-m) / k$ e $\lfloor a\rfloor$ representa o maior número inteiro igual ou menor que $a$. A distância média é calculada como $L(k)=\sum_{m=1}^{k} L_{m}(k) / k$ para $k=1, \ldots, k_{\max }$. A estimativa da FD, denotada por $F D_{H}$, é então dada pela inclinação da melhor aproximação linear de $\ln [L(k)]$ em função de $\ln (1 / k)$.
2) Algoritmo de Katz: Segundo [28], a FD de uma forma de onda $x(n)$ pode ser definida como

$$
F D_{K}=\frac{\log (L / a)}{\log (d / a)}
$$

onde $L$ é a soma das distâncias entre os pontos sucessivos de $x(n), a$ é a distância média entre os pontos sucessivos e $d$ é a maior distância entre $x(1)$ e os restantes pontos de $x(n)$.

\section{Energia}

A energia das sub-bandas está entre os parâmetros de EEG mais utilizados para detecção de DA e DP devido ao deslocamento energético das altas para as baixas frequências que ocorre nessas doenças. A energia de $x(n)$ é definida como

$$
E N=\sum_{n=1}^{N}|x(n)|^{2} \text {. }
$$

\section{E. Entropia}

A entropia é uma medida que descreve a quantidade de desordem, incerteza, em um sistema [22]. No caso de uma variável aleatória, a entropia quantifica a informação média associada às suas observações. As entropias de Shannon e Logarítimica podem ser estimadas como [29], [30]

$$
\begin{gathered}
E T_{S}=-\sum_{n=1}^{N}|x(n)|^{2} \log \left[|x(n)|^{2}\right] \\
E T_{L}=\sum_{n=1}^{N} \log \left[|x(n)|^{2}\right],
\end{gathered}
$$

respectivamente.

A entropia aproximada (ApET) é uma modificação na entropia de Kolmogorov-Sinai, a qual descreve a taxa na qual informações sobre o estado do sistema dinâmico são perdidas ao longo do tempo, para evitar divergências numéricas quando o sinal é contaminado por ruído, sendo definida como [22]

$$
\begin{aligned}
\operatorname{ApET}(m, r, N)= & \frac{1}{N-m+1} \sum_{i=1}^{N-m+1} \log \left[C_{i}^{m}(r)\right] \\
& -\frac{1}{N-m} \sum_{i=1}^{N-m} \log \left[C_{i}^{m+1}(r)\right],
\end{aligned}
$$

onde

$$
C_{i}^{m}(r)=\frac{1}{N-m+1} \sum_{j=1}^{N-m+1} \Theta\left(r-\left\|\mathbf{x}_{i}-\mathbf{x}_{j}\right\|\right)
$$

é a probabilidade do ponto $\mathbf{x}_{i}$ no atrator estar separado dos outros pontos por uma distância menor ou igual a $r$,

\section{Metodologia e Resultados}

Para cada um dos 32 indivíduos, os 10 parâmetros nãolineares $\left(D_{2}, L L E, \alpha, F D_{H}, F D_{K}, E N, E T_{S}, E T_{L} \mathrm{e}\right.$ $A p E T)$ foram calculados para as 5 sub-bandas $(\delta, \theta, \alpha, \beta$ e $\gamma$ ) do segmento médio e dos 20 segmentos de todos os 19 canais. E utilizados para discriminar, por sub-banda e canal, dentro dos seguintes pares de grupos de estudo: Controle vs DA, Controle vs DP, Controle vs DA/DP, DA vs DP. Ressaltase que os pacientes pertencentes aos grupos DA ou DP estão no estágio inicial MCI das doenças como descrito na Seção II. 


\section{A. Seleção da Wavelet}

Como os valores de cada parâmetro dependem da wavelet $\tilde{\psi}_{1}(n)$ utilizada na decomposição, uma busca foi realizada para encontrar a wavelet que resulta em parâmetros com maior capacidade discriminante para cada par de grupos de estudo. As famílias de wavelets avaliadas foram Biortognais, Biortogonais Reversas, Daubechies, Coiflets, Symlets e FejerKorovkin. E utilizou-se o segmento médio de cada indivíduo.

Os valores de cada parâmetro calculados a partir dos segmentos médios foram separados por cada combinação de par de grupos, wavelet, parâmetro e sub-banda. Em cada combinação, isso resulta em somente 1 valor (1 parâmetro de 1 sub-banda do segmento médio) por indivíduo. Dentro de cada combinação, os valores foram normalizados utilizando (1). E então, para cada combinação, os valores normalizados foram aplicados ao classificador binário X-ROC [31]. Esse classificador utiliza a distância quadrática entre médias de distribuições para encontrar um bom limiar para discriminar duas classes e a ROC (do inglês Receiver Operating Characteristic) para analisar o resultado da classificação [31].

Para cada par de grupos, selecionou-se a wavelet que resultou na maior quantidade de acurácias de classificação superiores a $80 \%$. As wavelets selecionadas foram Biortogonal Reversa 6.8, Biortogonal Reversa 3.5, Biortogonal Reversa 2.8 e Biortogonal 2.8 para os pares Controle vs DA, Controle vs DP, Controle vs DA/DP e DA vs DP, respectivamente. Ou seja, uma wavelet diferente foi utilizada em cada caso.

\section{B. Seleção de Parâmetros}

Como mencionado anteriormente, a discriminação dentro dos pares de grupos de estudo será realizada por sub-banda e canal. Assim, para cada combinação de sub-banda, canal e par de grupos, uma busca foi realizada para encontrar os parâmetros com maior capacidade discriminante. Para isso, utilizou-se os 20 segmentos de cada canal de cada indivíduo.

Os valores não-normalizados de cada parâmetro, calculados a partir de todos os segmentos de todos os canais, foram separados por cada combinação de par de grupos (e correspondente wavelet selecionada), sub-banda e canal. Em cada combinação, isso resulta em 200 valores de parâmetros (10 parâmetros obtidos de 1 sub-banda dos 20 segmentos de 1 canal) por indivíduo. Dentro de cada combinação, os valores foram normalizados utilizando (1). E então, para cada combinação, os valores normalizados foram aplicados a um algoritmo genético de forma a selecionar o melhor conjunto de 20 valores (10\% do total) por indivíduo.

\section{Classificação Utilizando Parâmetros Selecionados}

Para cada combinação de par de grupos de estudo e subbanda, o melhor conjunto de parâmetros foi utilizado como entrada de vários classificadores binários. Em todos os casos, para verificar a capacidade de generalização dos classificadores, uma validação cruzada leave-one-out foi utilizada. Os resultados do melhor classificador para cada caso estão indicados nos mapas topográficos exibidos na Figura 1, onde a acurácia máxima e o correspondente eletrodo estão destacadas.

$\mathrm{Na}$ discriminação entre os grupos patológicos, par DP vs DA, uma acurácia de $100 \%$ foi alcançada em todas as subbandas com exceção da $\gamma$, indicando uma efetiva capacidade discriminante dos parâmetros em todas as sub-bandas. Observa-se que essa acurácia ideal foi obtida por um grande número de eletrodos nas sub-bandas $\alpha$ e $\theta$, indicando que, nessas sub-bandas, os parâmetros analisados captam diferenças significativas entre as duas doenças por toda a cabeça. De forma geral, os elétrodos que alcançaram as maiores acurácias, acima de $87,5 \%$, estão localizados nas zonas parietal e central. E o classificador com melhor desempenho foi o SVM linear.

Em relação ao par DP vs Controle, uma acurácia de $100 \%$ foi alcançada nas sub-bandas $\gamma$ e $\delta$. Esses resultados foram obtidos através dos eletrodos localizados nas regiões frontal e parietal, as quais também foram apontadas como regiões de atividade da DP em [10]. Nas demais sub-bandas foram obtidas acurácias de classificação acima de $87,5 \%$. O classificador com melhor desempenho foi o método da regressão linear. As acurácias de $100 \%$ alcançadas nesse trabalho superam os resultados obtidos em [10], [11], os quais são inferiores a $90 \%$.

No estudo do par DA vs Controle, uma acurácia de $100 \%$ foi alcançada somente na sub-banda $\delta$. Nas outras sub-bandas foram obtidas acurácias entre $75 \%$ e $93,8 \%$. De forma geral, as regiões frontal e temporal mostraram ser o foco da atividade da DA como também indicado em [14]. O classificador com melhor desempenho foi a regressão logística. A acurácia de $100 \%$ alcançada nesse trabalho supera os resultados obtidos em [12], [13], [14], [15], os quais são inferiores a 94\%.

Por fim, o caso DA/DP vs Controle foi o único para o qual não se obteve nenhuma acurácia de $100 \%$. A maior acurácia de classificação foi $90,6 \%$ na sub-banda $\theta$. Nas demais subbandas foram obtidos resultados entre $68,8 \%$ e $87,5 \%$. As regiões de maior interesse são essencialmente a frontal e a temporal, onde são atingidos os valores máximos de acurácia. O classificador com melhor desempenho foi o SVM linear.

De forma geral, os resultados demonstraram que a combinação de parâmetros não-lineares multibanda de sinais EEG é promissora para detectar DA e DP em estágio inicial. No entanto, o número de exames EEG por grupo de estudo na base de dados utilizada é limitado e os resultados precisam ser atualizados com uma maior população para garantir a generalização. Além disso, o par DA/DP vs Controle é de particular interesse uma vez que permite detectar o aparecimento de ambas as doenças. $\mathrm{O}$ desempenho obtido indica que tal objetivo é um desafio e pesquisas adicionais são necessárias.

\section{CONClusÕes}

Este trabalho investigou a deteção das doenças de Alzheimer e Parkinson em estágio inicial através de parâmetros nãolineares multibanda de sinais EEG. A detecção foi realizada entre pares de grupos de estudo e a análise multibanda foi efetuada usando a transformada Wavelet.

Para cada par de grupos de estudo, uma seleção dos parâmetros foi realizada por sub-banda. Os parâmetros selecionados foram utilizadas como entrada para classificadores com validação cruzada leave-one-out. Acurácias de classificação de $100 \%$ foram obtidas, em pelo menos uma sub-banda, para os pares Controle vs DA, Controle vs DP e DA vs DP, enquanto $90,60 \%$ foi alcançado para o par Controle vs DA/DP. A subbanda $\delta$ foi a que, em geral, apresentou maiores diferenças significativas entre os grupos.

\section{AgRAdecimentos}

Os autores gostariam de agradecer à Unidade Neurológica do Hospital de São João, Porto, Portugal, pelo fornecimento da base de dados. Esse trabalho foi apoiado pelos Fundos Nacionais da Fundação para a Ciência e a Tecnologia (FCT), Portugal, através do projeto UIDB/50016/2020. 


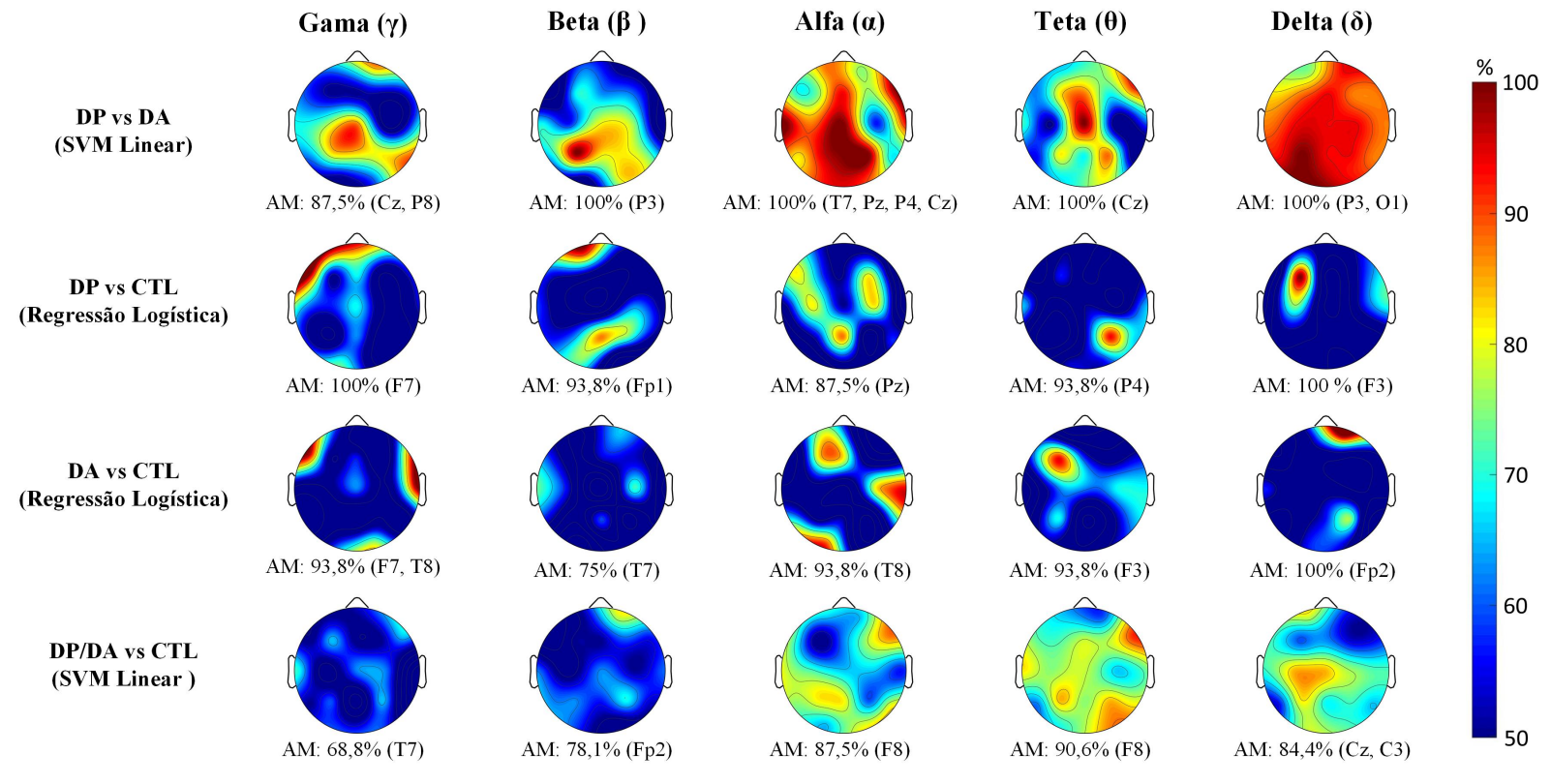

Fig. 1. Mapas topográficos das classificações obtidas entre pares de grupos de estudo ao nível dos eletrodos.

\section{REFERÊNCIAS}

[1] L. M. de Lau and M. M. Breteler, "Epidemiology of parkinson's disease," The Lancet Neurology, vol. 5, no. 6, pp. 525-535, June 2006.

[2] R. Mayeux and Y. Stern, "Epidemiology of alzheimer's disease," Cold Spring Harbor Perspectives in Medicine, vol. 2, no. 8, pp. 1-18, April 2012.

[3] R. Cassani, M. Estarellas, R. San-Martin, F. J. Fraga, and T. H. Falk, "Systematic review on resting-state EEG for alzheimer's disease diagnosis and progression assessment," Disease Markers, vol. 2018, pp. 1-26, October 2018.

[4] T. K. Khan, "An algorithm for preclinical diagnosis of alzheimer's disease," Frontiers in Neuroscience, vol. 12, April 2018.

[5] A. E. Lang and A. M. Lozano, "Parkinson's disease," New England Journal of Medicine, vol. 339, no. 15, pp. 1044-1053, October 1998.

[6] A. Valmarska, D. Miljkovic, M. Robnik-Sikonja, and N. Lavrac, "Visualization and analysis of parkinson's disease status and therapy patterns," in Discovery Science. Springer International Publishing, 2018, pp. 466479.

[7] A. Association, "Alzheimer's disease facts and figures 2018," Alzheimer's \& Dementia, vol. 14, no. 3, pp. 367-429, March 2018.

[8] N. Houmani, F. Vialatte, E. Gallego-Jutglà, G. Dreyfus, V.-H. NguyenMichel, J. Mariani, and K. Kinugawa, "Diagnosis of alzheimer's disease with electroencephalography in a differential framework," PLOS ONE, vol. 13, no. 3, p. e0193607, March 2018.

[9] M. Galea and M. Woodward, "Mini-mental state examination (MMSE)," Australian Journal of Physiotherapy, vol. 51, no. 3, p. 198, 2005.

[10] J. F. Cavanagh, P. Kumar, A. A. Mueller, S. P. Richardson, and A. Mueen, "Diminished EEG habituation to novel events effectively classifies parkinson's patients," Clinical Neurophysiology, vol. 129, no. 2, pp. 409418, February 2018.

[11] S. L. Oh, Y. Hagiwara, U. Raghavendra, R. Yuvaraj, N. Arunkumar, M. Murugappan, and U. R. Acharya, "A deep learning approach for parkinson's disease diagnosis from EEG signals," Neural Computing and Applications, August 2018.

[12] F. Vialatte, A. Cichocki, G. Dreyfus, T. Musha, S. Shishkin, and R. Gervais, "Early detection of alzheimer's disease by blind source separation, time frequency representation, and bump modeling of EEG signals," Lecture Notes in Computer Science, vol. 3696, pp. 683-692, 2005.

[13] G. Fiscon, E. Weitschek, G. Felici, P. Bertolazzi, S. D. Salvo, P. Bramanti, and M. C. D. Cola, "Alzheimer's disease patients classification through EEG signals processing," in 2014 IEEE Symposium on Computational Intelligence and Data Mining (CIDM). IEEE, December 2014.

[14] P. M. Rodrigues, D. R. Freitas, J. P. Teixeira, D. Alves, and C. Garrett, "Electroencephalogram signal analysis in alzheimer's disease early detection," International Journal of Reliable and Quality E-Healthcare, vol. 7, no. 1, pp. 40-59, January 2018.

[15] S. Khatun, B. I. Morshed, and G. M. Bidelman, "A single-channel EEG-based approach to detect mild cognitive impairment via speech- evoked brain responses," IEEE Transactions on Neural Systems and Rehabilitation Engineering, vol. 27, no. 5, pp. 1063-1070, May 2019.

[16] S. Sanei, EEG Signal Processing. Wiley-Blackwell, 2007.

[17] M. Vetterli and J. Kovačević, Wavelets and Subband Coding. Englewood Cliffs, New Jersey: Prentice Hall, 1995.

[18] H. S. Malvar, Signal Processing with Lapped Transforms. Norwood, Massachusetts: Artech House, 1992.

[19] O. Rioul and M. Vetterli, "Wavelets and signal processing," IEEE Signal Processing Magazine, vol. 8, no. 4, pp. 14-38, October 1991.

[20] A. BenSaïda, "A practical test for noisy chaotic dynamics," SoftwareX, vol. 3-4, pp. 1-5, December 2015.

[21] G. R. Bermúdez and P. J. G. Laencina, "Analysis of EEG signals using nonlinear dynamics and chaos - a review," Applied Mathematics \& Information Sciences, vol. 9, pp. 1-13, 012015.

[22] O. Faust and M. G. Bairy, "Nonlinear analysis of physiological signals: a review," Journal of Mechanics in Medicine and Biology, vol. 12, no. 04, p. 1240015, September 2012.

[23] C. J. Stam, "Nonlinear dynamical analysis of EEG and MEG: review of an emerging field," Clinical Neurophysiology, vol. 116, no. 10, pp. 1388-2457, October 2005.

[24] M. T. Rosenstein, J. J. Collins, and C. J. D. Lucaa, "A practical method for calculating largest Lyapunov exponents from small data sets," Physica D: Nonlinear Phenomena, vol. 65, no. 1, pp. 117-134, May 1993.

[25] B. Qian and K. Rasheed, "Hurst exponent and financial market predictability," in Proceedings of IASTED International Conference on Financial Engineering and Applications, Berkeley, USA, September 2007, pp. 203-209.

[26] P. Gifani, H. R. Rabiee, M. Hashemi, P. Taslimi, and M. Ghanbari, "Optimal fractal-scaling analysis of human EEG dynamic for depth of anesthesia quantification," Journal of the Franklin Institute, vol. 344, no. 3-4, pp. 212-229, 2007.

[27] J.-M. Lee, D.-J. Kim, I.-Y. Kim, K.-S. Park, and S. I. Kim, "Detrended fluctuation analysis of EEG in sleep apnea using MIT/BIH polysomnography data," Computers in biology and medicine, vol. 32, no. 1, pp. 37-47, 2002.

[28] M. J. Katz, "Fractals and the analysis of waveforms," Computers in Biology and Medicine, vol. 18, no. 3, pp. 145-156, January 1988.

[29] J. Rodríguez-Sotelo, A. Osorio-Forero, A. Jiménez-Rodríguez, D. Cuesta-Frau, E. Cirugeda-Roldán, and D. Peluffo, "Automatic sleep stages classification using EEG entropy features and unsupervised pattern analysis techniques," Entropy, vol. 16, no. 12, pp. 6573-6589, December 2014

[30] A. B. Das and M. I. H. Bhuiyan, "Discrimination and classification of focal and non-focal EEG signals using entropy-based features in the EMD-DWT domain," Biomedical Signal Processing and Control, vol. 29, pp. 11-21, August 2016.

[31] C. T. Nakas and C. T. Yiannoutsos, "Ordered multiple-class ROC analysis with continuous measurements," Statistics in Medicine, vol. 23, no. 22, pp. 3437-3449, November 2004. 